University of Wollongong

Research Online

Faculty of Engineering and Information

Faculty of Engineering and Information

Sciences - Papers: Part A

Sciences

$1-1-2013$

Synthesis and optimization of PEDOT:PSS based ink for printing

nanoarrays using Dip-Pen Nanolithography

Michal Wagner

Abo Akademi University

Cathal O'Connell

University of Wollongong, coc531@uowmail.edu.au

David G. Harman

University of Wollongong, dharman@uow.edu.au

Ryan Sullivan

University of Wollongong, rps759@uowmail.edu.au

Ari Ivaska

Abo Akademi University

See next page for additional authors

Follow this and additional works at: https://ro.uow.edu.au/eispapers

Part of the Engineering Commons, and the Science and Technology Studies Commons

Research Online is the open access institutional repository for the University of Wollongong. For further information contact the UOW Library: research-pubs@uow.edu.au 


\title{
Synthesis and optimization of PEDOT:PSS based ink for printing nanoarrays using Dip-Pen Nanolithography
}

\begin{abstract}
Due to the current interest in organic printable electronics, Dip-Pen Nanolithography (DPN) is increasingly being explored as a method to pattern electroactive materials such as conducting polymers (CPs) on the micro- and nanoscale. In general, printing process depends strongly on the ink-substrate properties and fundamental forces required to drive and stabilize the ink transfer to the substrate. Controlling these parameters is especially difficult when operating under the nanometer confinements of the DPN probe. For the printing of $\mathrm{CPs}$, one step towards addressing these challenges is rational ink design, as the use of existing commercial-based inks may not be suitable for the DPN ink transfer process on the nanoscale.
\end{abstract}

\section{Keywords}

ink, printing, optimization, nanoarrays, pedot, dip, pen, nanolithography, synthesis, pss

Disciplines

Engineering | Science and Technology Studies

\section{Publication Details}

Wagner, M., O'Connell, C., Harman, D. G., Sullivan, R., Ivaska, A., Higgins, M. J. \& Wallace, G. G. (2013). Synthesis and optimization of PEDOT:PSS based ink for printing nanoarrays using Dip-Pen Nanolithography. Synthetic Metals, 181 (1 October), 64-71.

\section{Authors}

Michal Wagner, Cathal O'Connell, David G. Harman, Ryan Sullivan, Ari Ivaska, Michael J. Higgins, and Gordon G. Wallace 


\title{
Synthesis and optimization of PEDOT:PSS based ink for printing nanoarrays using Dip-Pen Nanolithography
}

\author{
Michal Wagner, ${ }^{a}$ Cathal D. O’Connell, ${ }^{b}$ David G. Harman, ${ }^{b}$ Ari Ivaska, ${ }^{* a}$ Michael J. Higgins ${ }^{* b}$ and \\ Gordon G. Wallace ${ }^{b}$
}

\author{
${ }_{5}$ Received (in $\left.X X X, X X X\right) X$ th $X X X X X X X X X 20 X X$, Accepted Xth $X X X X X X X X X 20 X X$ \\ DOI: $10.1039 / b 000000 x$
}

Due the current interest in organic printable electronics, Dip-Pen Nanolithography (DPN) is increasingly being explored as a method to pattern electromaterials such as conducting polymers (CPs) on the microand nanoscale. In general, printing process depends strongly on the ink-substrate properties and

10 fundamental forces required to energetically drive and stabilize the ink transfer to the substrate.

Controlling these parameters is especially difficult when operating under the nanometer confinements of the DPN probe. For the printing of CPs, one step toward addressing these challenges is rational ink design, as the use of existing commercial-based inks may not be suitable for the DPN ink transfer process on the nanoscale. In this study, we synthesized and developed a poly(3,4 ethylenedioxythiophene)

15 poly(styrenesulfonate) (PEDOT:PSS)-based ink, to which we could add known constituents for optimizing the printing, adhesive and conductive properties of an aqueous dispersed ink. For silicon and gold surfaces, we demonstrate that the DPN pattering of the ink could achieve PEDOT:PSS (dot) arrays with diameters ranging from the submicron down to $\sim 160 \mathrm{~nm}$. These dimensions represent a dramatic improvement in resolution compared to previous attempts in patterning of CP via physioadsorption

${ }_{20}$ process using DPN. This work highlights that rational design of CP inks is critical, especially for DPN where the ink transfer process is governed by fluid physical properties and surface forces in the nanodomain. Knowledge of the constituents and overall ink composition will also lead to a greater understanding of these fundamentals that facilitate the pattering of CP inks on the nanoscale using DPN.

\section{Introduction}

${ }_{25}$ Conducting Polymers (CPs) are increasingly being integrated into microdevices due to their unique properties, including intrinsic ionic-electronic conductivity, mechanical flexibility and biofunctionality. Since CPs merge these attractive properties with "close to metals" electrical conductivities ${ }^{1}$, they are being 30 targeted for use as electronic components (e.g. electrode arrays) in bio-related applications such as biosensors, implantable electrodes and organic microelectronics. To gain an advantage, many of these technologies demand that the electronic components be scaled down and patterned in the nanodomain to 35 improve spatial resolution and signal-to-noise ratio. In particular, nanoscale CP sensors for detection of gases and biomolecules can provide relatively higher sensitivity and faster response times than conventional macro- and microsized sensors. ${ }^{2-5}$ Thus miniaturization of chemical and biological sensors is desirable 40 and provides further opportunities for lowering detection limits, reducing volumes needed for sensing, enhancing selectivity, increasing portability and in vivo applicability.

Fabrication of CPs at the nanoscale-level can be realized through template synthesis and post-assembly methods ${ }^{6,7}$ as well 45 as with use of photo- and soft lithography.,9 Template synthesis leads to formation of many kinds of discrete CP nanostructures but precisely controlling their position, for example as part of well-defined electrode array, is challenging. The nanoscale fabrication of CPs using conventional electrochemical deposition 50 is also difficult when applied to submicrometer or nanoscale dimensions due to deviations from simple mass transfer process (i.e., diffusive losses of monomer or reactants from the electrode). Top-down lithography approaches can be used to fabricate precise, high resolution nano-patterned structures from ${ }_{55}$ electrochemically prepared macro-sized films; however the use of "single-material" films limits the composition diversity of the patterned structures and areas. Lithography methods have been extensively used for patterning of $\mathrm{CPs}^{10}$ and especially microcontact printing that can offer versatile, low-cost, surface 60 patterning and submicron accuracy. ${ }^{11}$ For microcontact printing, an elastomeric stamp is covered with ink and placed in contact against a substrate and in one step it is possible to print a whole series of patterns. Some applications require a process termed "multiplexing” whereby different inks are simultaneously printed. ${ }_{65}$ One technique that is able to complement other lithography techniques through multiplexing whilst also patterning with nanoscale resolution is Dip-Pen Nanolithography (DPN). DPN is based on the transfer of ink onto the substrate by a tip with nanoscale dimensions, usually in the form of an atomic force 70 microscopy (AFM) cantilever tip. ${ }^{12}$ DPN has been used for the 
multiplex printing of different proteins, or protein deposition within existing patterns. ${ }^{13,14}$ DPN has been extensively used for the printing of metal nanoparticle, ${ }^{15-17}$ organic compounds ${ }^{12,18,19}$ and more recently applied to patterning of CPs. ${ }^{20-24}$

5 The DPN deposition process is ascribed by two mechanisms depending of the type of material that is deposited. When patterning relatively small molecules the transport through a liquid meniscus has been proposed. ${ }^{12,25}$ The spontaneous formation of a meniscus between the tip and substrate, which is 10 dependent on the surrounding humidity, facilitates molecular diffusion. ${ }^{26}$ For liquid-based inks, the deposition is strongly dependent on liquid-surface interactions and generally driven by physio-adsorption processes. ${ }^{27}$ Liquid inks can importantly act as a "carrier" for different types of particulates or biological 15 molecules. ${ }^{28}$ In the case of CP dispersions, the ink properties are governed by the CP aggregate-size and related molecular weight and chemistry of the polymer. The major challenges in the application of CP dispersions in DPN printing appear to be connected to dispersion instability, effects of solvent evaporation 20 and adhesion to the substrate. ${ }^{24}$

Herein we concentrated on printing the conducting poly(3,4 ethylenedioxythiophene) poly(styrenesulfonate) (PEDOT:PSS). The patterning of PEDOT is of special interest due to its attractive physical and chemical properties. PEDOT is thermally 25 and electrochemically stable with relatively low redox potentials. ${ }^{29,30}$ When doped with PSS surfactant, it also highly solution processable in comparison with other conducting polymers. ${ }^{30}$ Importantly, PEDOT:PSS is a favourable material for biological studies due to its biocompatibility. ${ }^{31}$ PEDOT:PSS 30 water dispersions have previously been used as “inks" in DPN studies and deposited as lines ${ }^{20}$ and dot patterns. ${ }^{24}$ In the latter approach, commercially available PEDOT:PSS dispersions have been concentrated and used as received. This included an assessment of the DPN printing of a commercial PEDOT:PSS ink 35 on different types of surfaces, including hard and flexible substrates. ${ }^{24}$ However, these commercial inks are specifically designed for their intended use in spin coating and/or ink-jet printing and may not necessarily be optimized for the transfer mechanisms, or nanometer confined volumes, governing the 40 operation of DPN. For example, solvent evaporation and particulate aggregation is a major issue when relying on the transfer of such small volumes (attolitres), while the nanoscale forces involved in successful transfer of ink are easily influenced (or often adversely affected) by the ink properties, surface 45 chemistry and environmental conditions. We recently found that a commercial PEDOT:PSS could not be reproducibly used for printing as received. ${ }^{24}$ Stable printing of arrays composed of dots $\sim 1 \mu \mathrm{m}$ in diameter, with the smallest dimensions of $\sim 700-800$ nm, was only achieved through modification of the ink with 50 addition of methanol. Although further optimization of the commercial ink was desired in this study (i.e. to reduce the dot dimension and solvent evaporation effects), it was not possible as the composition of the commercial ink was unknown.

In this study, we aimed to develop a PEDOT:PSS ink starting 55 from raw material synthesised in our labs and then through the incorporation of different additives, such as thickeners, surfactants and ion exchangers. In nanolithography polyalcohols such as glycerol and poly(ethylene glycol) were successfully used e.g. to improve the mobility of the ink on the polymer pens in 60 mixtures of proteins and buffer solutions ${ }^{32}$ or to assist photoresist microfabrication. ${ }^{33,34}$ Hence, herein, the main additive to our PEDOT:PSS inks was glycerol that had the added function of improving the conductivity of the bulk PEDOT:PSS. Applied modifications provided a more rational approach toward the 65 development an ink and allowed various ink properties to be systematically varied for optimization of the DPN process.

\section{Experimental}

\subsection{Materials and PEDOT:PSS synthesis}

The basic ink additives: glycerol, hexadecyltrimethylammonium 70 bromide (CTAB) and Triton X-100 were purchased from SigmaAldrich. Commercial PEDOT:PSS was Orgacon Dry dispersible pellets (Orgacon ${ }^{\mathrm{TM}}$ Electronic Materials, AGFA). The custom synthesized PEDOT:PSS was made with the use of catalyst in the following way. NaPSS of 70,000 MW (4.954g, $24.02 \mathrm{mmol}$ of 75 repeating unit, 4.421 g PSS equiv, 2.06 mass ratio) was dissolved in $150 \mathrm{~mL}$ of water. Ammonium persulfate (4.455g, 19.52 mmol) was added to the stirred solution which was then deoxygenated with a flow of $\mathrm{N}_{2}$ bubbles for $5 \mathrm{~min}$. The solution was stirred at $750 \mathrm{rpm}$ then EDOT $(1.5 \mathrm{~mL}, 2.145 \mathrm{~g}$, $8015.09 \mathrm{mmol}$ ) was added by syringe in one shot. A few crystals of $\mathrm{Fe}_{2}\left(\mathrm{SO}_{4}\right)_{3} \cdot 9 \mathrm{H}_{2} \mathrm{O}\left(1 \mathrm{mg}, 210^{-6} \mathrm{~mol}\right)$ were added as a catalyst. The solution was stirred under nitrogen at room temperature for 20 hours, at which time a thick deep blue colored paste was evident. The mixture was transferred to a centrifuge tube with 85 the aid of $50 \mathrm{~mL} \mathrm{H}_{2} \mathrm{O}$ and spun at $4500 \mathrm{rpm}$ for $30 \mathrm{~min}$, and the supernatant was decanted. Addition of water (135 mL) centrifugation (4500rpm/30min) and decantation wasrepeated another 4times. Then $500 \mathrm{~mL}$ of water was added and the mixture was placed in an ultrasound bath for $60 \mathrm{~min}$, followed by 90 centrifugation and decantation. Another $500 \mathrm{~mL}$ of water was added and the mixture was boiled for 2 hours, cooled and then centrifuged once more. The supernatant was drawn off and the final water was removed by rotary evaporation under reduced pressure. The final estimated yield was $70-80 \%$. The specific 95 conductivity of the material was $36 \pm 2 \mathrm{~S} \mathrm{~cm}^{-1}$ untreated and 141 $\pm 7 \mathrm{~S} \mathrm{~cm}^{-1}$ when the drop-cast film was treated with ethylene glycol and the material heated at $120^{\circ} \mathrm{C}$ for $30 \mathrm{~min}$.

\subsection{Ink preparation}

Both commercial and custom synthesized PEDOT:PSS were 100 dissolved in ultrapure water ( $2.5 \%$ solid content). The dispersions were left for 5 minutes in a sonication bath followed by an extensive tip sonicaton for 10 minutes and finally transferred into a high-speed homoganizer. After plain PEDOT:PSS solutions were obtained, tip sonication was repeated 105 to ensure that the PEDOT:PSS solutions could be passed through a $1.2 \mu \mathrm{m}$ pore size syringe filter. For preparation of inks ready to print using DPN, 0.2 volume \% of Triton X-100 was added to the sonicated and filtered PEDOT:PSS dispersions. CTAB with a concentration of $0.01 \mathrm{M}$ was dissolved in glycerol and assisted 110 with heating. The final ink formulation was prepared by mixing the PEDOT:PSS dispersion containing Triton X-100 with 10 volume \% of glycerol (+ $0.01 \mathrm{M} \mathrm{CTAB})$ and then used immediately for DPN printing. Conductivity and viscosity measurements were made using four point probe (Jandel RM3- 
AR) and rheometer (TA Instruments AR-G2) respectively.

\subsection{DPN deposition and characterization of patterned PEDOT:PSS inks}

DPN printing was performed using an Nscriptor system and 5 controlled by InkCAD ${ }^{\mathrm{TM}}$ software (both provided by Nanoink Inc.). Twelve pen tip-arrays (Type $\mathrm{M}$, A-frame cantilevers with spring constant of $0.6 \mathrm{~N} \mathrm{~m}^{-1}$, made of $\mathrm{Si}_{3} \mathrm{~N}_{4}, 107 \mu \mathrm{m}$ in length and $22 \mu \mathrm{m}$ in width) were used for printing and silicon inkwell arrays were used for inking. Silicon substrates were also obtained 10 from Nanoink Inc. Gold surfaces were prepared on silicon by evaporation of $4 \mathrm{~nm}$ thick layer of chromium and $16 \mathrm{~nm}$ layer of gold. The substrates before printing were rinsed with ultrapure water. During the DPN process, the temperature and humidity were kept at $22 \pm 3{ }^{\circ} \mathrm{C}$ and $65 \%$, respectively. Imaging was 15 conducted using an Asylum Research MFP-3D AFM instrument (Asylum Research, Santa Barbara, CA) and Wyko NT9100 Optical Profilometer (Bruker, Germany). In order to determine an "optimal" volume \% of added glycerol in the PEDOT:PSS ink, the glycerol content was adjusted from $3 \%$ to $25 \%$. After the 20 addition of each volume \% glycerol, the inks were assessed for DPN printing. For water stability tests, the substrates with printed patterns were annealed at $105{ }^{\circ} \mathrm{C}$ for $8 \mathrm{~h}$. Glycerol containing $0.01 \mathrm{M}$ of cetrimonium bromide (CTAB) was then drop casted on the patterned areas and left for $24 \mathrm{~h}$. After soaking, the samples

25 were washed with methanol and annealed again using the same conditions as above.

\section{Results and discussion}

\subsection{Preparation of PEDOT:PSS material}

PEDOT:PSS starting material was obtained via two different 30 sources. We used a custom synthesized PEDOT:PSS (herein referred to PEDOT:PSS CS, see experimental methods for synthesis) tailored for obtaining relatively high conductivities and, for comparison, a commercially available Orgacon Dry PEDOT:PSS material that was selected because to the best of our 35 knowledge did not contain any surfactant or additives.

After dispersing the two different types of PEDOT:PSS in water through extensive sonication, their physical properties were assessed and are listed in Table 1. PEDOT:PSS CS has both longer PEDOT and PSS chains compared to the commercial 40 material, in addition to a tenfold higher viscosity. In order to deliver as much as possible of CP material to the sample surface we used relatively higher solid content ( 2.5 \%) of PEDOT:PSS aqueous dispersions whencompared to typical commercial dispersions (e.g. up to $1.3 \%$ of solid content).

45 Table 1 shows that conductivity of both PEDOT:PSS inks as drop-casted films increased significantly with the additives (see Table 2) in comparison to PEDOT:PSS without any additives. It is believed that the increase in the electrical conductivity of the PEDOT:PSS is related to an optimal polymer structure, or 50 secondary doping mechanism, when the glycerol is added. This is analogous to previously reported effects on the inclusion of polyalcohols into PEDOT:PSS films that increase their electrical conductivity. ${ }^{35}$

\subsection{Additives and rational ink development}

${ }_{55}$ Printing of PEDOT:PSS with DPN directly from a pure aqueous dispersion is problematic mainly due to dispersion instability and solvent evaporation. ${ }^{24}$ Thus to develop a suitable ink the additives listed in Table 2 were incorporated into both the custom synthesized and commercial PEDOT:PSS to rationally address 60 the intended properties and functions of the ink. Among the important physical properties of the ink is a low surface tension and vapour pressure. In particular, proper wetting of the tip is essential during the process of sufficiently "inking” the AFM tip when it (the tip) is dipped into microfluidic wells. To lower the 65 surface tension of the aqueous PEDOT:PSS, the surfactant Triton $\mathrm{X}-100$ was added. Another consideration of the ink properties is that the high volatility of water modifies the ink properties over time and subsequent lack of stability may prevent or temporally limit patterning or upscalability of the printing process. To 70 address this aspect, the addition of glycerol greatly improves ink stability and, as already discussed, also results in an increase in the PEDOT:PSS conductivity. After systematically adjusting the glycerol concentration followed by assessment for DPN patterning, we found that $10 \%$ was the minimal concentration 75 required to enable PEDOT:PSS patterns (i.e. no patterns observed below this value). The addition of higher glycerol \% showed no apparent improvement in printing since the obtained patterns qualitatively looked the same. The use of these additives follows similar studies that have investigated the use of glycerol and 80 Triton- $X$ in commercial aqueous PEDOT:PSS inks for inkjet printing. ${ }^{36}$ However, the composition of these commercial PEDOT:PSS ink, which most likely contains other constituents, are unknown. Patterning of PEDOT:PSS from solutions containing only one additive (Triton X-100 or glycerol) did not 85 give any printing results even at higher concentrations.

The addition of the quaternary ammonium salt, cetrimonium bromide (CTAB), was intended to have a twofold function. $\mathrm{CTAB}$ is a cation surfactant and acts as a second dispersant in the ink. Its other desired function is related to the improvement of the 90 PEDOT:PSS stability and adhesion to the substrate after the CP patterns had been immersed in water or electrolyte. PEDOT:PSS in water forms particles in which the core consists of PEDOT and the outer-layers of negatively charged PSS groups. The interaction between PEDOT:PSS particles can therefore be 95 stabilized by the addition of cations, which in some case results in the formation of a gel. Multivalent cations such $\mathrm{Mg}^{2+}, \mathrm{Ca}^{2+}$ or $\mathrm{Ru}\left(\mathrm{NH}_{3}\right)_{6}{ }^{2+/ 3+}$ have previously been used to crosslink PEDOT:PSS. ${ }^{37}$ The addition of ammonium cation also results in crosslinking of PEDOT:PSS particles. For example, $\mathrm{CTA}^{+}$ 100 exchanges with sodium and hydrogen ions and because the CTA ${ }^{+}$ is relatively large the "crosslinking effect" is enhanced. ${ }^{38}$ The deposition of PEDOT:PSS with this ammonium salt was seen as a means to improve stability through enhanced cross-linking and possible surface adhesion of the deposited PEDOT:PSS patterns 105 after exposure to an aqueous solution, which is required for their electrochemical applications. The concentration of CTAB in glycerol was adjusted to $0.01 \mathrm{M}$ and was generally the highest concentration at which ease of solubility could be achieved. Printing with the inks containing both Triton X-100 and glycerol 110 but without CTAB is possible, though the addition of the ammonium salt greatly prolonged the printing times before needing to re-ink the tips in the silicon inkwell arrays. This was most likely due to the increased stability of the PEDOT:PSS 
dispersion.

\subsection{DPN pattering of PEDOT:PSS inks}

In order to demonstrate the deposition and patterning of the PEDOT:PSS based inks, multi-pen tips (twelve pens) were used 5 in the DPN for printing. For each experiment, the multi-pen tips were dipped into inkwells and then initial contact made with the surface prior to patterning to remove excess ink from the tip. After this initial "bleeding" step, it was possible to stably pattern dots across all 12 pens for well in excess of over an hour, which 10 amounted to $40 \mu \mathrm{m}$ dot arrays (comprising 9x9 dots) being able to cover an approximate surface area of $2 \times 2 \mathrm{~mm}$ (each pen is separated by $\sim 60 \mu \mathrm{m})$. Fig. $1 \mathrm{~A}$ and B shows optical profilometer images of patterned regions from 3 different pens for the different PEDOT:PSS inks. Some variation in dot dimensions within, and 15 between arrays, were observed, particularly the first dot printed in each array and subsequent dots along the first row (i.e. bottom row of arrays). Non-uniformities in the dot size may largely be a consequence of utilizing a liquid based ink. For example, care must be taken when comparing the results achievable by liquid 20 inks vs molecular inks. The rate of transport of molecular inks (e.g. MHA on gold) can be regarded as constant over a short timeframe. However, in the case of liquid inks accumulation of the ink on the end of the tip can occur with time, resulting in a larger than average dot at the beginning of a grid and 25 subsequently along the first row. Registration of the dots relative to the original array pattern inputted in the InkCAD ${ }^{\mathrm{TM}}$ software was not always precise. In contrast to a molecular ink that will adsorb directly on to the substrate at the point of deposition, it is likely that the precise location adopted by a liquid ink droplet, is 30 subject to the phenomenon of contact line pinning (due to, for example, surface energy inhomogeneity, surface roughness, surface defects, the dynamics of deposition etc.) The result is that the deposited liquid feature will sometimes migrate slightly to adopt a lower energy arrangement on the surface.

35 Further imaging of the arrays using AFM for a more detailed analysis was performed on individual 9x9 dot arrays for both PEDOT:PSS inks (commercial and customized PEDOT:PSS ink) on gold and silicon substrates using a dwell time of one second for the DPN process (Fig. 2). The patterned arrays and 40 dimensions of individual dots showed good uniformity, with individual dots diameters having sub-micron dimensions. A statistical analysis of the dot dimensions was performed by measuring the height section profiles of individual dots. For example, from the representative AFM images presented in Fig. 452 , 25 dots were arbitrary chosen for analysis. We specifically made the analysis for a region of dots ( $3 \times 3=9$ dots) in smaller scan areas of $10 \mu \mathrm{m}$ to alleviate the effect of tip broadening and confirmed values using smaller scans of $1 \mu \mathrm{m}$ to image single dots (randomly selected). All measured dimensions were 50 performed from several AFM images and summarized in Table 3. The measured values of both dot dimensions of height and diameter exhibits a very narrow distribution of dimensions for each ink, indicating that the DPN deposition process provided uniform patterning, particularly within arrays. It was found that 55 all inks routinely gave dot widths less than $1 \mu \mathrm{m}$, with the customized PEDOT:PSS ink achieving significantly reduced widths down to $200 \mathrm{~nm}$ in diameter, as shown in Fig. 2F of single dot of the customized PEDOT:PSS ink on silicon. Given an expected deviation (over-estimation) due to tip broadening 60 effects, these latter dimensions are comparable with DPN printing of poly (ethylene glycol) based block copolymer on silicon ${ }^{39}$ and mixtures of agarose and tricine on glass substrates. ${ }^{28}$ The standard deviation of the dot dimensions indicates the degree of uniformity and reproducibility of the patterning, which is also 65 similar to that reported during DPN printing of thiols and acid monolayers deposited on gold. ${ }^{40}$ The dot heights were all lower than $50 \mathrm{~nm}$, indicating a relatively small aspect ratio (dot height/width) of the deposited features. The calculated aspect ratio for dots patterned from the customized PEDOT:PSS ink is 70 around tenfold higher compared to the commercial PEDOT:PSS.

The size of printed dots depends strongly on the contact dwell time of the AFM tip at the surface. To asses this effect and perhaps gain more insight into the ink properties, dwell time calibration patterns were printed using both PEDOT:PSS inks on 75 gold and silicon. For these calibrations, analysis of the dimensions were carried out on $1 \mathrm{x} 1 \mu \mathrm{m}$ images of single dots totalling 20 dots. Fig. 3 presents an example of AFM images from the dot size calibration and single images of each dot along the same row. The measured dimensions were plotted as a 80 function of the dwell time ${ }^{1 / 2}$ and showed a linear relationship (Fig. 4), as previously observed for diffusion-controlled processes of ink deposition using DPN. For dot width, the commercial ink showed a stronger dependency (greater slope) on dwell time, indicating an effect from differences in the ink chemistries of the 85 raw PEDOT:PSS material. In contrast, there were no difference in dwell-time dependencies between the different material substrates, gold and silicon, for both inks. For dot height, the dependencies were similar for both inks, with the calibration again confirming previous analysis that the dot heights were 90 clearly greatest for the customized PEDOT:PSS on silicon. These observations confirmed that the dot widths were greater overall for the commercial ink, while the customized PEDOT:PSS ink provided higher aspect ratio dimensions and made it possible to achieve the smallest dimensions of $\sim 160 \mathrm{~nm}$ dot width when 95 applying very fast dwell times of $0.1 \mathrm{~s}$. We do note that in some cases variations in the mean dot dimensions, particularly the width, were observed between arrays or dots produced from different pens. For example, we observed differences in mean dot widths between arrays (Table 3) and those of the calibration 100 patterns (Fig. 4) for the commercial ink on silicon and CS-based ink on gold at $1 \mathrm{~s}$ dwell times. These differences may relate to the variation in dot sizes observed in Fig. 1A and B and also correlate with our previous multi-array DPN patterning of a commercial PEDOT:PSS ink that showed a distribution of dot dimensions 105 within an array of a single pen, and also between pens, both of which could vary by $10-15 \%{ }^{24}$

Chemically analysing the components of the final dot printed features is difficult due to the nanoscale dimensions of the dots. Previous studies on AFM-based liquid deposition have 110 shown that the evaporation rate of micron-sized glycerol dots occurs within minutes and is extremely rapid for similar attolitre volumes used in this study ${ }^{41}$. With the addition of annealing the patterns at $105^{\circ}$, we expect that the final component will contain little glycerol. Closer inspection of the AFM images, particularly 115 for the gold substrate, show that the printed dots are actually made up of individual particles of $\sim 50-100 \mathrm{~nm}$, suggesting the 
presence of PEDOT:PSS nanoparticles (Fig. 2E). Aqueous dispersions of PEDOT:PSS are known to consist of nanoparticles ${ }^{42}$, and the presence and size of these nanoparticles in our PEDOT:PSS dispersions were confirmed by zetasizer 5 measurements (data not shown).

Similarly, measuring the conductivity of individual dots is difficult due to their nanoscale dimensions. We have previously been able to use conductive AFM imaging and AFM-based I-V spectroscopy to qualitatively show conductive properties of $\sim 1$ $10 \mu \mathrm{m}$ commercial PEDOT:PSS dots patterned via $\mathrm{DPN}^{24}$, however, quantifying their conductivity values was not straightforward using these approaches. To overcome this, it is possible to quantify conductivity from lines patterned between two electrodes, as done in our previous work on vapour phase 15 polymerized PEDOT:PSS patterned via $\mathrm{DPN}^{23}$. Unfortunately, the patterning of lines using the PEDOT:PSS inks in this study was problematic and did not allow for quantifying the conductivity. As the ink is liquid based, deposition occurs upon break of contact between the tip and surface (as opposed to 20 molecular ink transport, where deposition occurs via continuous diffusion). We suggest that the successful drawing of nano- and microscale lines of liquid would require a delicate balance of capillary forces (e.g. liquid surface tension, substrate surface energy), or else the engineering of a chemical affinity between 25 the ink and substrate.

\subsection{Water resistance}

Water stability of PEDOT:PSS was improved by introducing 10 volume \% of glycerol to which $0.01 \mathrm{M}$ of CTAB was dissolved. 30 After printing, the patterned arrays were annealed at $105{ }^{\circ} \mathrm{C}$ for several hours. The patterns were then soaked again in $0.01 \mathrm{M}$ $\mathrm{CTAB}$ in glycerol to perform another ion exchange. After washing away excess of glycerol from patterned arrays using methanol, the samples were annealed again at $105{ }^{\circ} \mathrm{C}$. This 35 process offered improved adhesion of PEDOT:PSS to the surface in a water environment. Fig. 5 show an example of commercial PEDOT:PSS patterns printed on silicon after the treatment and after having being exposed to water for up to 2 hours. When tested on the bulk scale (e.g. films with surface area of $0.07 \mathrm{~cm}^{2}$ ), 40 the deposited ink treated in this way could survive in water for up to one month (data not shown). This treatment process was found to be critical, as annealing itself was not sufficient to provide the enhanced adhesion and stability in water. Furthermore, we could not obtain any water resistance of the patterned arrays, as well as 45 of bulk films of PEDOT:PSS, without addition of CTAB. The exact mechanism of the improved stability and adhesion is unclear though both ion exchange and annealing may be responsible. For ion exchange, exchanging with sodium and hydrogen cation and interacting with PSS chains may limit the 50 solubility of the PEDOT:PSS particles and/or directly crosslink them. However, the annealing and CTAB treatment process did not work for the PEDOT:PSS CS-based ink. This may be due to the PSS chains in PEDOT:PSS CS being longer and thus are more easily dispersed than in the commercial material. To utilize 55 the electrochemical properties of these patterned arrays, their stability in aqueous media is necessary and through the addition of salt exchangers it is feasible to enhance stability/adhesion; however further studies on the implementation of adhesion promoters are required to optimize the process.

\section{4. Conclusions}

By synthesizing and rationalizing designing a new PEDOT:PSS ink, we have been able to achieve pattern dimensions that are much smaller than the limit expected for a liquid ink transferred via physioadsorption processes, which is typically on the order of ${ }_{65} \sim 0.5$ to $1 \mu \mathrm{m}$. The minimum dot widths down to $\sim 160 \mathrm{~nm}$ represent a significant reduction in dimension size for these types of liquid inks. This ability was afforded by the use of additives to enable DPN printing of an aqueous dispersed PEDOT:PSS material, increased adhesion of the ink to the substrate, and 70 enhancement of the conductivity. Furthermore, knowledge of the individual constituents and overall ink composition is critical for enabling systematic studies on the effects of the polymer-dopant mass ratio and different additives - this will lead to a greater understanding of the fluid physical properties that facilitate the 75 pattering of conducting polymer inks on the nanoscale using DPN. The ability to pattern liquid conducting polymers inks on such small scales present interesting possibilities for various applications, including conducting polymer-based sensors, organic and flexible electronics on the nanoscale and bionics 80 applications where biological moieties could conceivably be incorporated into the ink.

\section{Acknowledgments}

Financial support from the Finnish Graduate School of Materials Research (GSMR) and Australian National Fabrication Facility ${ }_{85}$ (ANFF) is gratefully acknowledged. This work is a part of activities at Åbo Akademi Process Chemistry Centre, within the Finnish Centre of Excellence Programme (2000-2011) of the Academy of Finland. The authors thank Dr. Hiroshi Nakashima for many valuable discussions.

\section{${ }_{90}$ Notes and references}

${ }^{a}$ Process Chemistry Centre, c/o Laboratory of Analytical Chemistry, Åbo Akademi University, Biskopsgatan 8, FI-20500, Turku, Finland. Fax: +358 2215 4479; Tel: +358 2215 4420;

E-mail: aivaska@abo.fi

$9_{95}^{b}$ ARC Centre of Excellence for Electromaterials Science, Intelligent

Polymer Research Institute, Innovation Campus, University of

Wollongong, Wollongong, NSW 2522, Australia. Fax: +61 (2) 4221

3114; Tel: +61 (2) 4221 3989;

E-mail:mhiggins@uow.edu.au

100

1 A.J. Heeger, Synth. Met., 2001, 125, 3.

2 M.H. Yun, N.V. Myung, R.P. Vasquez, C. Lee, E. Menke and R.M. Penner, Nano Lett., 2004, 4, 419.

3 H.Q. Liu, J. Kameoka, D.A. Czaplewski and H.G. Craighead, Nano 105 Lett., 2004, 4, 671.

4 K. Ramanathan, M.A. Bangar, M.H. Yun, W. Chen, N.V. Myung and A. Mulchandani, J. Am. Chem. Soc., 2005, 127, 496.

5 J.X. Huang, S. Virji, B.H. Weiller and R.B. Kaner, J. Am. Chem. Soc., 2003, 125, 314.

1106 L. Pan, H. Qiu, C. Dou, Y. Li, J. Xu and Y. Shi, Int. J. Mol. Sci., 2010, 11, 2636.

7 K. Jackowska, A.T. Biegunski and M. Tagowska, J. Solid State. Electrochem., 2008, 12, 437.

8 G.M. Wallraff and W.D. Hinsberg, Chem. Rev., 1999, 99, 1801. 
9 Y. Xia and G.M. Whitesides, Angew. Chem. Int. Ed., 1998, 37, 550.

10 C. Luo, Microsyst. Technol., 2009, 15, 1605.

11 A. Perl, D.N. Reinhoudt and J. Huskens, Adv. Mater., 2009, 21, 2257.

12 R.D. Piner, J. Zhu, F. Xu, S. Hong and C.A. Mirkin, Science, 1999, 283, 661.

13 K.-B. Lee, J.-H. Lim and C.A. Mirkin, J. Am. Chem. Soc., 2003, 125, 5588.

14 J.-H. Lim, D.S. Ginger, K.-B. Lee, J. Heo, J.-M. Nam and C.A. Mirkin, Angew. Chem. Int. Ed., 2003, 42, 2309.

1015 W.K. Lee, Z. Dai, W.P. King and P.E. Sheehan, Nano Lett., 2010, 10, 129.

16 S.-C. Hung, O.A. Nafday, J.R. Haaheim, F. Ren, G.C. Chi and S.J. Pearton, J. Phys. Chem. C, 2010, 114, 9672.

17 B. Wu, A. Ho, N. Moldovan and H.D. Espinosa, Langmuir, 2007, 23, 85 159120.

18 K. Salaita, Y. Wang, J. Fragala, R.A. Vega, C. Liu and C.A. Mirkin, Angew. Chem. Int. Ed., 2006, 45, 7220.

19 J.-W. Jang, A. Smetana and P. Stiles, Scanning, 2010, 32, 24.

20 H.-H. Lu, C.-Y. Lin, T.-C. Hsiao, Y.-Y. Fang, K.-C. Ho, D. Yang,

20 C.-K. Lee, S.-M. Hsu and C.-W. Lin, Anal. Chim. Acta, 2009, 640, 68.

21 J.-H. Lim and C.A. Mirkin, Adv. Mater., 2002, 14, 1474.

22 M. Yang, P.E. Sheehan, W.P. King and L.J. Whitman, J. Am. Chem. Soc., 2006, 128, 6774.

2523 C. O'Connell, M.J. Higgins, H. Nakashima, S.E. Moulton and G.G. Wallace, "Vapor phase polymerization of EDOT from submicron scale oxidant patterned by dip-pen nanolithography" (submitted, under review)

24 H. Nakashima, M.J. Higgins, C. O’Connell, K. Torimitsu and G.G. Wallace, Langmuir, 2012, 28, 804.

25 J. Haaheim, R. Eby, M. Nelson, J. Fragala, B. Rosner, H. Zhang and G. Athas, Ultramicroscopy, 2005, 103, 117.

26 B.L. Weeks, M.W. Vaughn and J.J. DeYoreo, Langmuir, 2005, 21, 8096.

3527 H.-T. Wang, O.A. Nafday, J.R. Haaheim, E. Tevaarwerk, N.A. Amro, R.G. Sanedrin, C.-Y. Chang, F. Ren and S.J. Pearton, Appl. Phys. Lett., 2008, 93, 143105.

28 A.J. Senesi, D.I. Rozkiewicz, D.N. Reinhoudt and C.A. Mirkin, ACS Nano, 2009, 3, 2394.

4029 J.W. Schultze and H. Karabulut, Electrochim. Acta, 2005, 50, 1739.

30 L. Groenendaal, F. Jonas, D. Freitag, H. Pielartzik and J.R. Reynolds, Adv. Mater., 2000, 12, 481.

31 M. Berggren and A. Richter-Dahlfors, Adv. Mater., 2007, 19, 3201.

32 Z. Zheng, W.L. Daniel, L.R. Giam, F. Huo, A.J. Senesi, G.Zheng, 115

45 and C.A. Mirkin, Angew. Chem. Int. Ed., 2009, 48, 7626.

33 J.-W. Jang, R.G. Sanedrin, A.J. Senesi, Z.Zheng, X. Chen, S. Hwang, L. Huang, and C.A. Mirkin, Small, 2009, 5, 1850.

34 J.-W. Jang, Z. Zheng, O.-S. Lee, W. Shim, G. Zheng, G.C. Schatz and C.A. Mirkin, Nano Lett., 2010, 10, 4399.

5035 A.M. Nardes, R.A.J. Janssen and M. Kemerink, Adv. Funct. Mater., 2008, 18, 865.

36 F. Ely, C.O. Avellanenda, P. Paredez, V.C. Nogueira, T.E.A. Santos, V.P. Mammana, C. Molina, J. Burg, G. Gibson and L. Zhao, Synth. Met., 2011, 161, 2129.

5537 M. Vázquez, P. Danielsson, J. Bobacka, A. Lewenstam and A. Ivaska, Sens. Actuators B, 2004, 97, 182.

38 M. Döbbelin, R. Marcilla, C. Tollan, J.A. Pomposo, J.-R. Sarasua and D. Mecerreyes, J. Mater. Chem., 2008, 18, 5354.

39 J. Chai, F. Huo, Z. Zheng, L.R. Giam, W. Shim and C.A. Mirkin, 130

60 Proc. Natl. Acad. Sci. U.S.A., 2010, 107, 47.

40 S. Rozhok, R. Piner and C.A. Mirkin, J. Phys. Chem. B, 2003, 107, 751.

41 T. Ondarcuhu, J. Arcamone, A. Fang, H. Durou, E. Dujardin, G. Ruis, and F. Perez-Murano, Eur. Phys. J. Special Topics, 2009, 166, 135 $65 \quad 15-20$.

42 J. Pecher and S. Mecking, Chem Rev. 2010, 110, 6260-6279. 
Table 1 Physical properties of studied PEDOT:PSS based inks and their deposits.

\begin{tabular}{|c|c|c|c|c|}
\hline Ink type & $\begin{array}{l}\text { Solid } \\
\text { content/\% }\end{array}$ & Viscosity/Pa s & $\begin{array}{c}\text { Conductivity }{ }^{\mathrm{a}} \mathrm{S} \\
\mathrm{cm}^{-1} \\
\text { (additives) }\end{array}$ & $\begin{array}{c}\text { Conductivity } / \mathrm{S} \\
\mathrm{cm}^{-1} \\
\text { (without } \\
\text { additives) }\end{array}$ \\
\hline $\begin{array}{c}\text { Commercial } \\
\text { PEDOT:PSS } \\
\text { based ink } \\
\text { (Orgacon } \\
\text { Dry) }\end{array}$ & $\sim 2.5$ & $\sim 0.017$ & $142 \pm 30$ & $13.0 \pm 0.7$ \\
\hline $\begin{array}{c}\text { PEDOT:PSS } \\
\text { CS based ink } \\
\text { (custom } \\
\text { synthesized) }\end{array}$ & $\sim 2.5$ & $\sim 0.178$ & $114 \pm 58$ & $1.4 \pm 0.2$ \\
\hline
\end{tabular}


25 Table 2 List of ink additives.

\begin{tabular}{|c|c|c|}
\hline Ink constituents & Concentration & Function of additive \\
\hline Triton X-100 & 0.2 volume $\%$ & Improve wetting \\
\hline Glycerol & 10 volume $\%$ & $\begin{array}{l}\text { Ink stability, } \\
\text { conductivity increase }\end{array}$ \\
\hline CTAB & $0.01 \mathrm{M}$ & $\begin{array}{l}\text { Dispersibility, cation } \\
\text { exchange (adhesion) }\end{array}$ \\
\hline
\end{tabular}




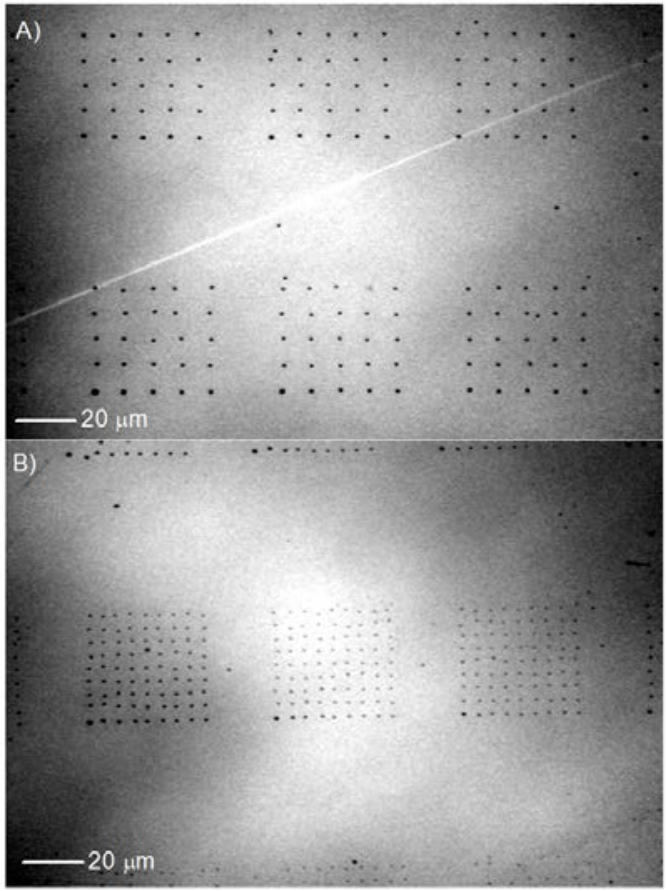

Fig. 1 An example of optical profilometer images of regions of patterned PEDOT:PSS on silicon substrates A) and B) are PEDOT:PSS dot-arrays containing 25 and 81 dots respectively within $40 \times 40 \mu \mathrm{m}$. 

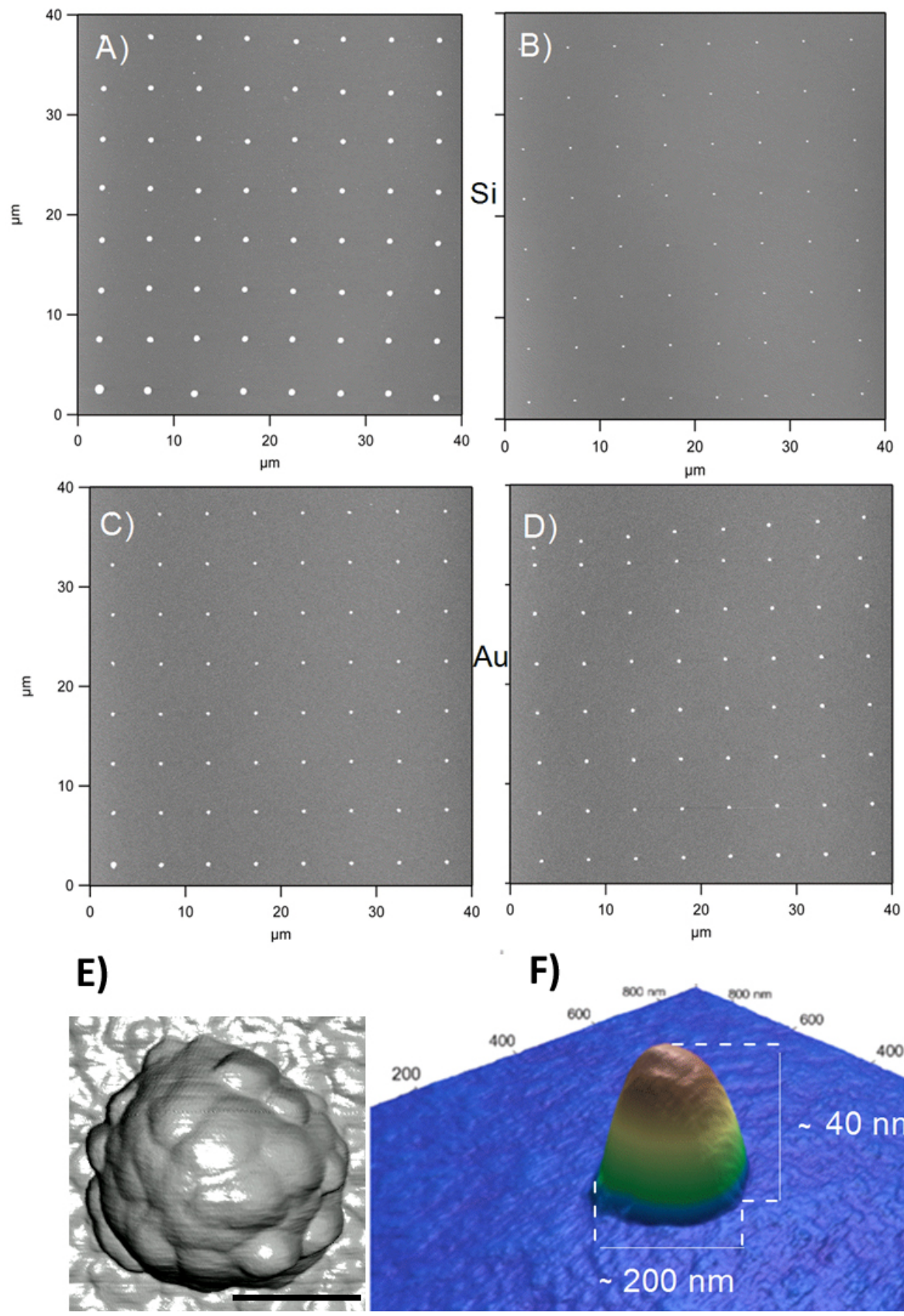

F)

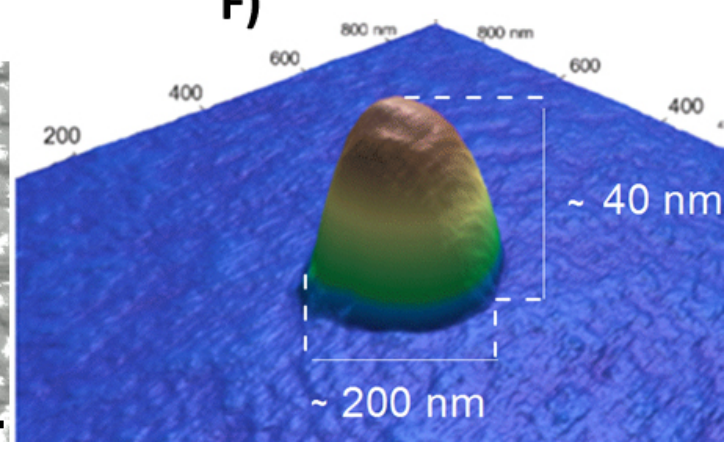

${ }_{5}$ Fig. 2 AFM topographic images of PEDOT:PSS 81 dot patterns printed on silicon (A, B) and gold (C, D) from commercial PEDOT:PSS based ink (A, C) and PEDOT:PSS CS based ink (B, D). The dots were patterned by multi-pen with dwell time of one second and spacing between dots is $\sim 5 \mu \mathrm{m}$. High magnification 3-D topographic images of a single dot of customized PEDOT:PSS on gold (E) and (F) silicon. Scale bar in (E) is $190 \mathrm{~nm}$ 
Table 3 The dot size of the PEDOT:PSS dots patterned on gold and silicon measured using height section profiles from AFM images.

\begin{tabular}{|c|c|c|c|c|}
\hline Ink type & Substrate & Dot width/nm & Dot height/nm & Aspect ratio ${ }^{a}$ \\
\hline $\begin{array}{l}\text { commercial } \\
\text { PEDOT:PSS }\end{array}$ & $\mathrm{Si}$ & $641 \pm 10$ & $17 \pm 1$ & 0.03 \\
\hline based ink & $\mathrm{Au}$ & $399 \pm 5$ & $16 \pm 1$ & 0.04 \\
\hline $\begin{array}{c}\text { PEDOT:PSS CS } \\
\text { based ink }\end{array}$ & $\mathrm{Si}$ & $203 \pm 2$ & $28 \pm 1$ & 0.14 \\
\hline
\end{tabular}

20 


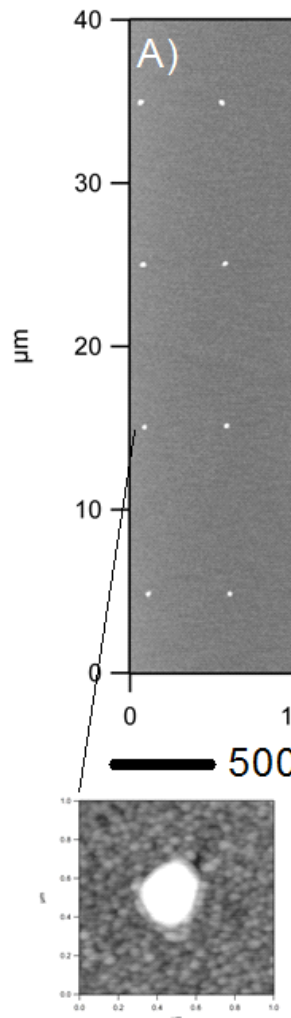

$0.01 \mathrm{~s}$

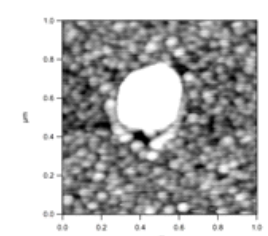

$0.1 \mathrm{~s}$

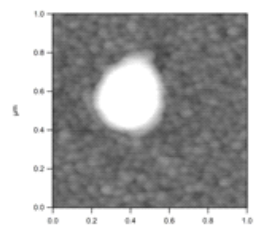

$0.5 \mathrm{~s}$

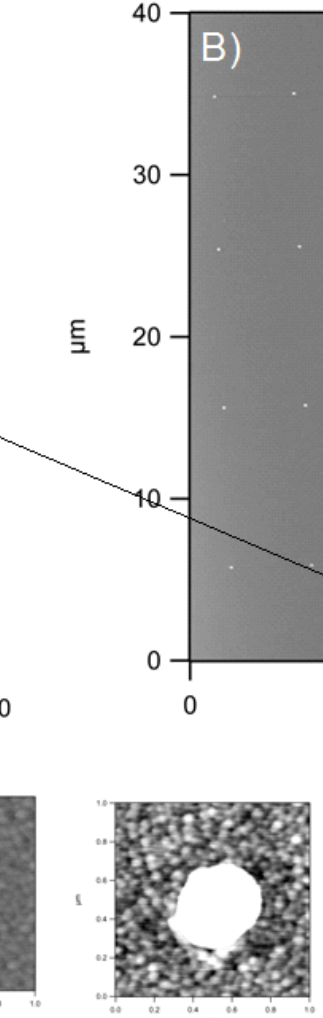

$1 \mathrm{~s}$

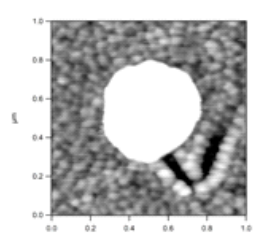

$2 \mathrm{~s}$

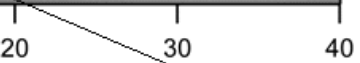

$\mu \mathrm{m}$

Fig. 3 AFM topographic images of dot size calibration for pattern printed on gold form commercial PEDOT:PSS based ink (A) and on silicon from PEDOT:PSS CS based ink (B). Insert: an example of AFM topographic images $(1 \mathrm{x} 1 \mu \mathrm{m})$ of each dot in the calibration pattern. 


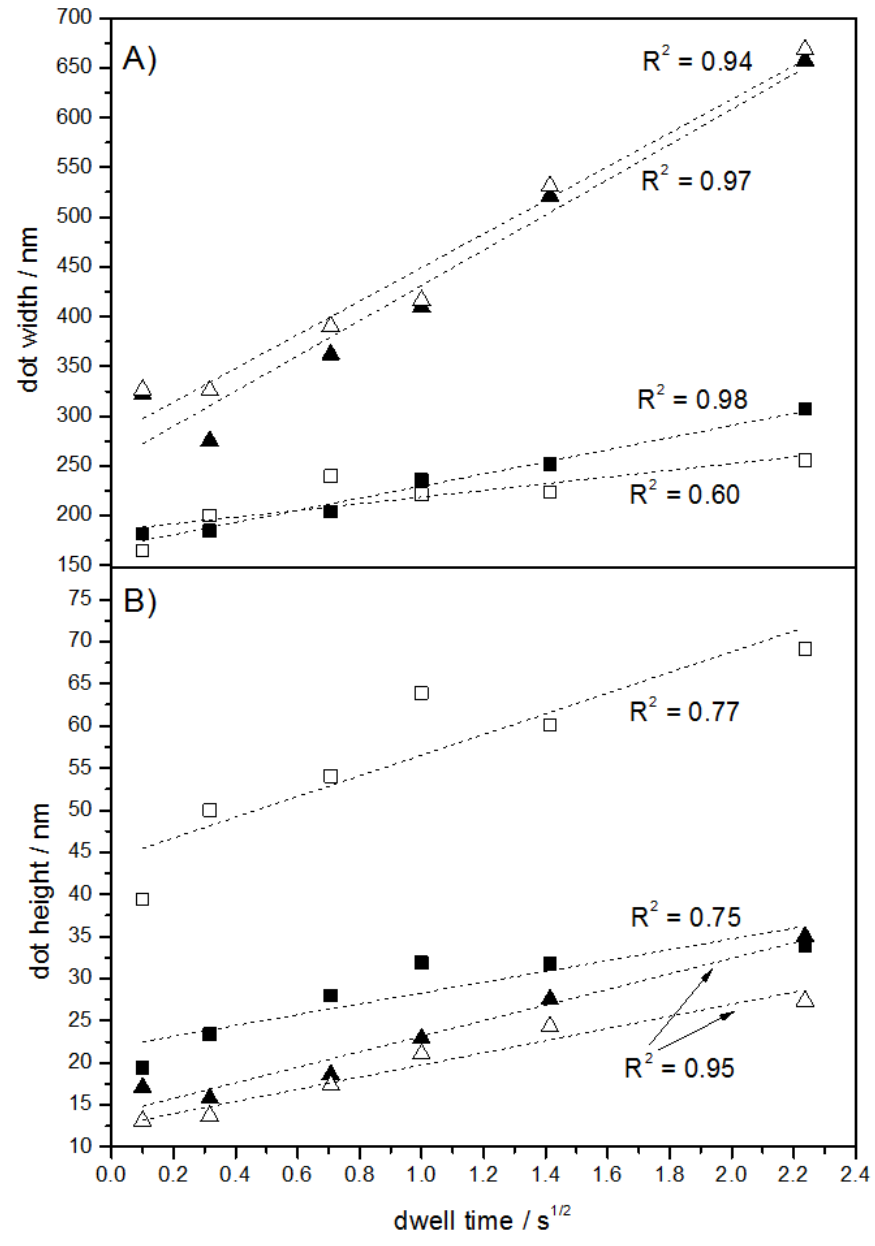

Fig. 4 Dots widths (A) and heights (B) plotted as a function of the dwell time. Representation: PEDOT:PSS CS on Si (ש) and Au (口) together with commercial PEDOT:PSS on $\mathrm{Si}(\boldsymbol{\Delta})$ and $\mathrm{Au}(\Delta)$. 


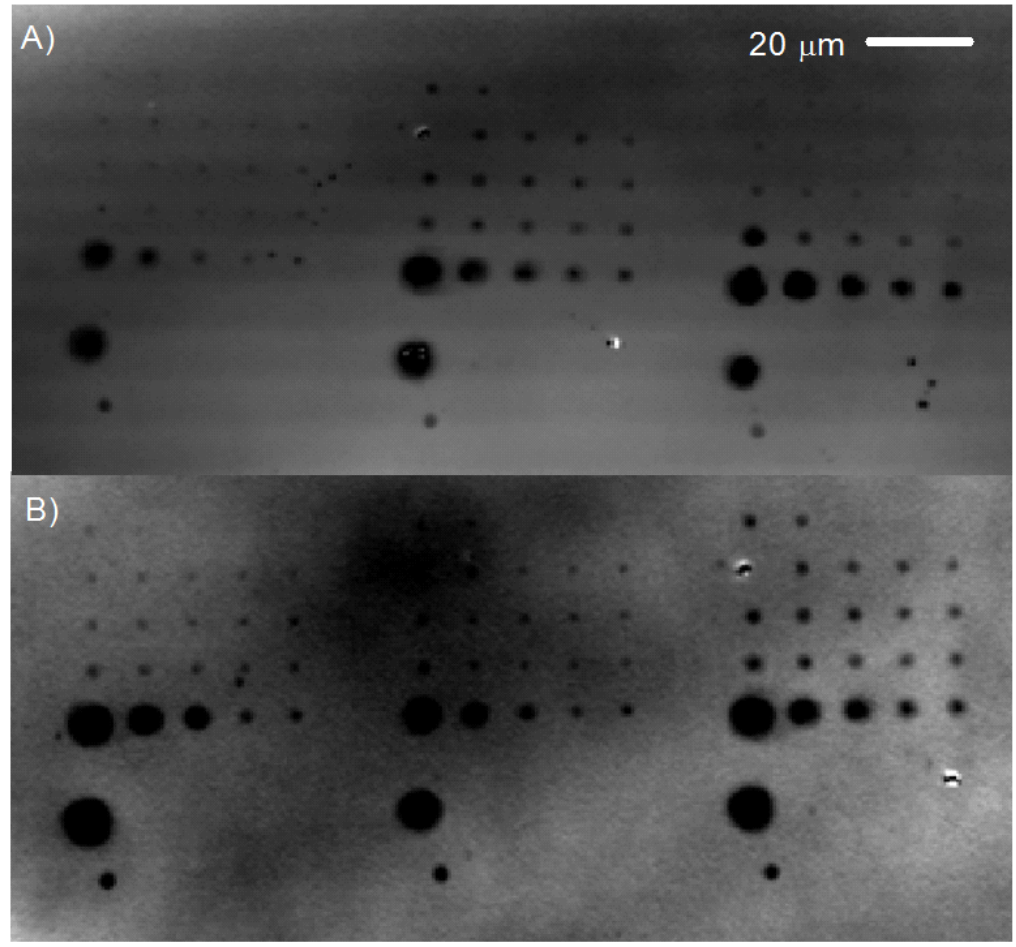

Fig. 5 Optical profilometer images of PEDOT:PSS arrays on silicon after soaking for 24 hours in $0.01 \mathrm{M}$ CTAB in glycerol followed by cleaning in methanol (A) and after exposure to water for 2 hours (B). 


\section{Table of content (TOC)}

In-house synthetized PEDOT:PSS was used in production of ink which includes several additives making printing process by Dip-Pen 5 Nanolithography suitable for obtaining conducting polymer nanoarrays which can be applied in biology and chemical sensors technology. We report that with our inks one can obtain feature sizes of 160-200 nm.

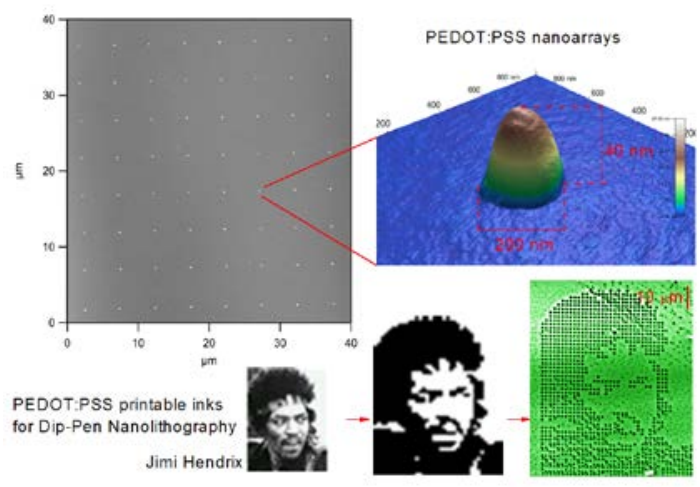

Picture for table of content entry 